Math. Model. Nat. Phenom.

Vol. 2, No. 1, 2008, pp. 81-103

\title{
Cell Modelling of Hematopoiesis
}

\author{
N. Bessonov ${ }^{a}$, L. Pujo-Menjouet ${ }^{b 1}$ and V. Volpert ${ }^{b}$ \\ ${ }^{a}$ Institute of Mechanical Engineering Problems, 199178 Saint Petersburg, Russia \\ ${ }^{b}$ Université de Lyon, Université Lyon1, CNRS UMR 5208 Institut Camille Jordan \\ F - 69200 Villeurbanne Cedex, France
}

\begin{abstract}
In this work, we introduce a new software created to study hematopoiesis at the cell population level with the individually based approach. It can be used as an interface between theoretical works on population dynamics and experimental observations. We show that this software can be useful to study some features of normal hematopoiesis as well as some blood diseases such as myelogenous leukemia. It is also possible to simulate cell communication and the formation of cell colonies in the bone marrow.
\end{abstract}

Key words: hematopoiesis modelling, multi-agent, leukemia, stem cell, bone marrow, cell competition.

AMS subject classification: 92K05, 92C37, 92C17, 92D25, 90C15

\section{Introduction}

\subsection{Origin of the problem}

Modeling cell proliferation related to blood cell formation (hematopoiesis) has been widely investigated for the past decades. Experimental and theoretical studies have been proceeded in different scales from a molecular level to a cell to cell contact and finally to a whole population dynamics. Some of these works were done in order to understand the mechanisms that determine proliferation, differentiation and apoptosis of blood cells as well as their reaction when facing various blood diseases like anemia, leukemia, neutropenia and others. Several attempts have been proposed to study blood cell population and blood diseases. Amongst the first attempts one can refer to the work initiated by Lajtha [20] and Burns and Tannock [9] where the authors included a resting phase in their

\footnotetext{
${ }^{1}$ Corresponding author. E-mail: pujo@math.univ-lyon.fr
} 
model. The most recent models in blood diseases in the literature are the so-called maturity-agetime structured models consisting of a system of partial differential equations describing instability of the cell population facing aplastic anemia $[3,4,13,14]$. Some other deterministic models using either systems of differential equations or age structured systems deal with cyclical neutropenia $[5,6,16,17]$, thrombocytopenia [25, 26], or chronic myelogenous leukemia [1, 2, 10, 22, 23, 24]. It is important here to notice that most of the continuous models deal with density only while our approach in the paper is to consider a population of individual cells. This allows us to describe their behavior and interactions in a more explicit way. On the other hand, it requires a more specific information about the regulatory networks that determine cell proliferation, differentiation and apoptosis. The software developed in the framework of this approach can be considered as an interface that can be adapted to various normal and pathological situations. We believe that it can also help analyzing biological experiments and medical data.

The paper is organized as follows. In the next section we remind briefly the biological background motivating our work. In section 3 , we simulate the normal and leukemic hematopoiesis. In section 4, we introduce and illustrate the problem of cell communication and give a conclusion. Note that we concentrate ourselves into the software presentation in this present paper. Thus for a better clarity, we decided to put the details of the construction of the model into the appendix.

\subsection{Biological background}

Blood cell formation also called hematopoiesis occurs mainly in the bone marrow. It is a process in which three main cell types are produced and regulated: the red blood cells (or erythrocytes), the white blood cells (or leukocytes) and the platelets (or thrombocytes). The function of each type is well known and understood now. Their daily production is fairly high: each second for instance, the body produces 2 millions of erythrocytes, also 2 millions of thrombocytes and 700, 000 granulocytes. Their lifetime differs from one type to another (120 days for erythrocytes, about 7 to 10 for the thrombocytes, and 6 to 14 hours only for the granulocytes (the shortest lifetime of these cell types). For the white cells, two different branches appear: lymphoid and myeloid. Note that we do not consider the lymphoid branch since all the diseases we investigate here do originate from the myeloid one. Then, it is now commonly accepted that all these blood cells originate from a single cell type: the hematopoietic stem cells (HSC). However, from the best of our knowledge, the density, lifetime and frequency of division of the HSC remain unclear. Indeed, identification of the HSC is still "an uncertain business and must rely on indirect measures since their morphological characteristics are unknown", see Mackey [21]. After dividing, the HSC can give birth to the so called progenitor cells. These cells divide into precursor cells giving birth to the 3 main lineages (see Figure 1 (myeloid lineage)).

Once in a specific lineage, a cell can not go back to an undifferentiated type. A precursor cell is committed to differentiate to the next cell type of the same branch. So, once a cell is being differentiated its fate is quite restricted: it can divide and give birth to two daughter cells with a higher maturity, it can differentiate, it can rest for a certain time that can be its lifetime, creating a reservoir in case of blood loss, or it can die by apoptosis (natural cell death).

At this point, three biological aspects should be discussed. Indeed, it has been shown (see 


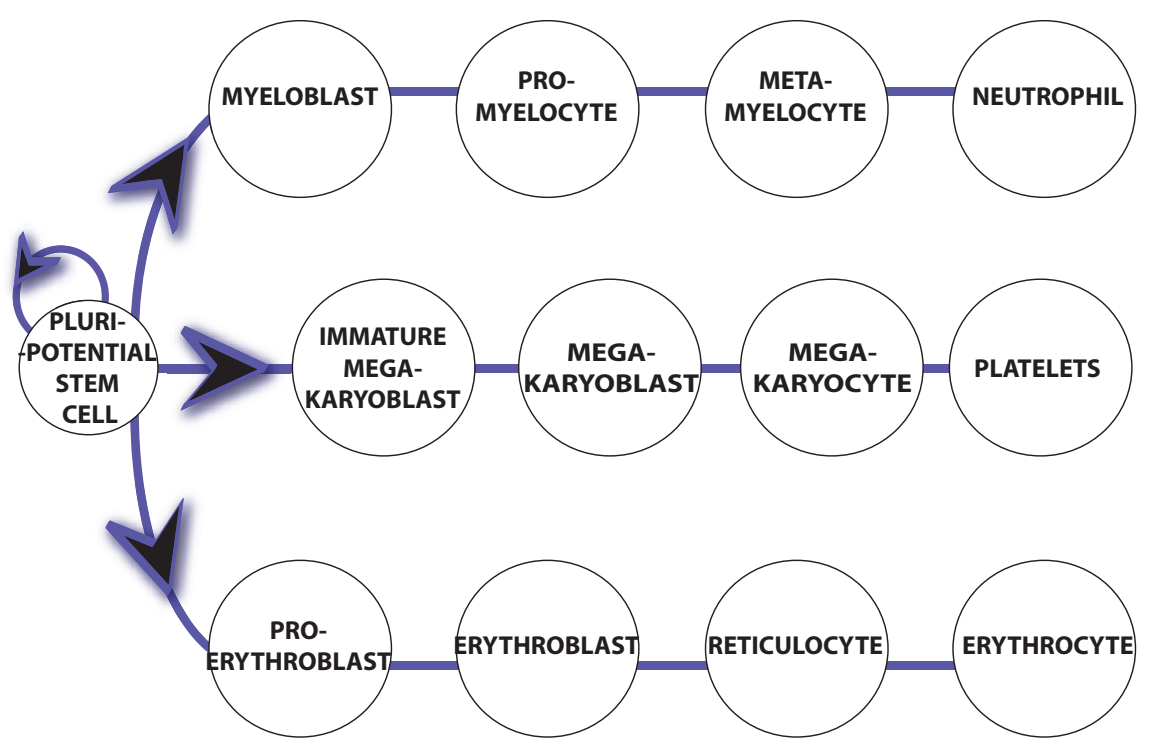

Figure 1: Scheme of the myeloid lineage formation (red blood cells, platelets and neutrophil cells). A stem cell can divide into either two other stem cells to renew the initial density in case of blood renewal, or one stem cell and one of the three cell types detailed above.

$[11,12])$ that some progenitor cells can be self renewable under hormone stimulations such as cortisol. We do not take this into account here but we are currently working on it. The second and third notions deal with two hypothesis that are still under investigations. One is about the fact that some mature cells can show reversible differentiation under some circumstances. To the best of our knowledge, this has been observed only in the intestinal crypt cells but not in the bone marrow. This kind of behavior could be simulated easily with our software, but since our focus is the blood cell formation, we decided not to develop this aspect here. The other hypothesis concerns cell migration. Some senescent blood cells are believed to migrate back to the bone marrow for some unclear reasons. Our software could be able to illustrate this with some slight modifications in our model. However, we think that a strong feedback with biologists is necessary to solve this question. This aspect is currently under discussion. Nevertheless, we claim that our model and thus our software has the potential strength to be adapted depending on the cell types, spatial configuration as well as some specific circumstances. Not everything can be shown here, but each point mentioned above is the object of our attention, and will appear in our future work.

Here, we assume that a stem cell can divide into either two other stem cells to renew the initial density in case of blood renewal, or one stem cell and one of the three cell types detailed above [27]. This scenario is shown in figure 2 but the user of our software can set up the rules differently depending on the experiment to simulate. In the next section let us introduce then a good illustration of our software which is the case of normal and leukemic hematopoiesis. Before this, we invite the reader to look at the appendix to get an insight of the model as well as a simple 


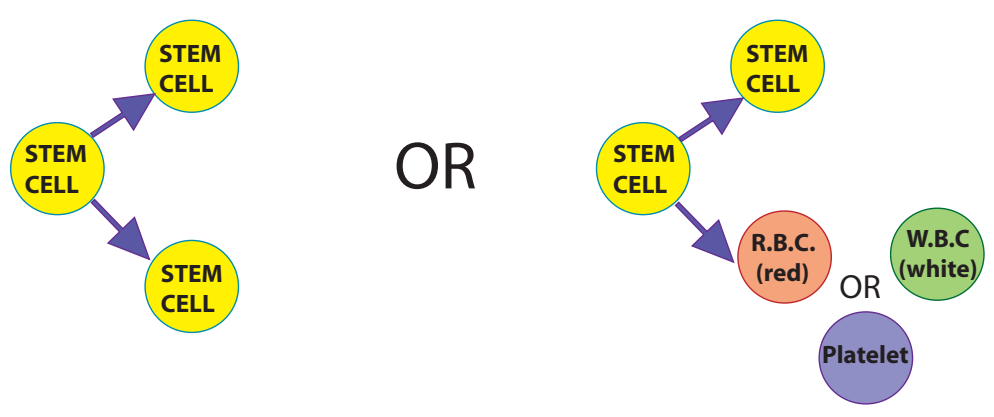

Figure 2: Scheme of stem cell division. A stem cell can divide into either two other stem cells to renew the initial density in case of blood renewal, or one stem cell and one of the three cell types detailed above. Note that our software is not restricted to this case.

example. Moreover, the software manual as well as the code is available upon request to the authors.

\section{Normal and leukemic hematopoiesis}

We begin our modeling of hematopoiesis with the scheme shown in the table in figure 3 . It describes the myeloid branch seen in figure 1:

$$
\begin{gathered}
A 0 \rightarrow A 0+B 1+E 1+F 1, \quad B 1 \rightarrow B 2 \rightarrow B 3 \rightarrow B 4, \quad E 1 \rightarrow C 1+D 1, \\
C 1 \rightarrow C 2 \rightarrow C 3 \rightarrow C 4, \quad D 1 \rightarrow D 2 \rightarrow D 3 \rightarrow D 4, \quad F 1 \rightarrow F 2 \rightarrow F 3 \rightarrow F 4 .
\end{gathered}
$$

Yellow cells $A 0$ are considered as stem cells, they are attached to the left boundary. They are selfrenewable, and produce three other cell types, $B 1, E 1, F 1$. The first step $A 0 \rightarrow A 0+B 1+E 1+F 1$ corresponds to a simplified description of the scheme $A 0 \rightarrow A 0+B 1, \quad A 0 \rightarrow A 0+E 1, \quad A 0 \rightarrow$ $A 0+F 1, \quad A 0 \rightarrow A 0+A 0$. Here, a stem cell gives four daughter cells at once instead of dividing four times. We understand that it is not biologically realistic to consider such a behavior, but it gives an equivalent qualitative behavior. Which is our first insight. On the other hand, we believe that it could be quite interesting to introduce stochasticity at the stem division level : a stem cell could give an offspring of a different type with a certain probability. This has not been coded yet but will be a part of our future work.

In figure 3, proliferation of the stem cells occurs every $10 \pm 2$ time units, proliferation of $B 1$ cells each $200 \pm 40$ time units (the parameters given here are not experimental values). Differentiation of cells in the $C$-, $D$-, $E$-, and $F$-lineages also occurs every $200 \pm 40$ time units. The life time of $C 4, D 4, E 4$, and $F 4$ cells (the most mature cells) is chosen very large $\left(10^{20}\right.$ time units) in order to be considered infinite for simplicity of the simulation with no loss of generality. It is clear in this example that only the most mature cells exit the computational domain through the 


\section{Normal hematopoiesis : myeloid lineage}

\begin{tabular}{|c|c|c|c|c|c|c|c|c|}
\hline New & & & & & & \multicolumn{3}{|r|}{ ? $x$} \\
\hline Parent & Chld1 & Chld2 & Chld3 & Chld4 & Time of. & $1+1-\mathrm{Ti}_{\mathrm{i}}$ & \begin{tabular}{l|l} 
iim... & Radius. \\
\end{tabular} & Ia \\
\hline$\checkmark \quad 40$ & $\mathrm{~A}_{0}$ & B1 & E1 & $\mathrm{F} 1$ & 10 & 2 & 0.01 & \\
\hline$\checkmark \mathrm{B} 1$ & B2 & . & . & . & 200 & 40 & 0.01 & \\
\hline$\sqrt{12}$ & 83 & . & . & & 200 & 40 & 0.01 & \\
\hline$\sqrt{83}$ & 84 & & & . & 200 & 40 & 0.01 & \\
\hline$\checkmark \quad$ B4 & & . & & . & $1 e+020$ & 2 & 0.01 & \\
\hline$\checkmark$ E1 & $\mathrm{Cl}$ & D1 & & . & 200 & 40 & 0.01 & \\
\hline$\checkmark \mathrm{Cl}$ & $\mathrm{C} 2$ & . & - & & 200 & 40 & 0.01 & \\
\hline$\checkmark \mathrm{C} 2$ & C3 & - & - & . & 200 & 40. & 0.01 & \\
\hline$\checkmark \quad$ c3 & C4 & & & . & 200 & 40 & 0.01 & \\
\hline$\checkmark \quad C 4$ & - & & & . & $1 e+020$ & 2 & 0.01 & \\
\hline$\checkmark$ D1 & D2 & & & . & 200 & 40 & 0.01 & \\
\hline$\checkmark \mathrm{D} 2$ & D3 & . & - & & 200 & 40 & 0.01 & \\
\hline$\checkmark \quad D 3$ & D4 & - & & . & 200 & 40 & 0.01 & \\
\hline$\checkmark \quad D 4$ & & . & . & . & $1 e+020$ & 2 & 0.01 & \\
\hline & $\mathrm{F} 2$ & . & . & . & 200 & 40 & 0.01 & \\
\hline$F 2$ & F3 & & & . & 200 & 40 & 0.01 & 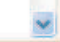 \\
\hline \multicolumn{2}{|l|}{$x=0.4$} & \multicolumn{3}{|c|}{$\operatorname{visc}(0 . .1)=0.2$} & $10=$ & & $F_{\max }=1$ & \\
\hline \multicolumn{2}{|l|}{$y=0.5$} & & ensity $(0 . \ldots 1)=$ & $=0.2$ & $g 0=$ & 0 & $F$ min $=\sqrt{0}$ & \\
\hline \multicolumn{2}{|c|}{ New area } & & Apply & \multirow{2}{*}{\multicolumn{3}{|c|}{$\begin{array}{l}\text { Obstacle coordinates: } \\
\text { Xmin }=0.05\end{array}$}} & & \\
\hline & & & & & & & $x_{\max }=0.06$ & \\
\hline \multicolumn{4}{|c|}{ V $f=a 4, g=0 / f=0, g=b 4$} & & min $=\sqrt{0.03}$ & & $Y_{\max }=0.07$ & \\
\hline \multicolumn{4}{|c|}{ PartofTimeLife $(0 . .1)=0.9$} & & $\ulcorner$ obsta & & & \\
\hline
\end{tabular}
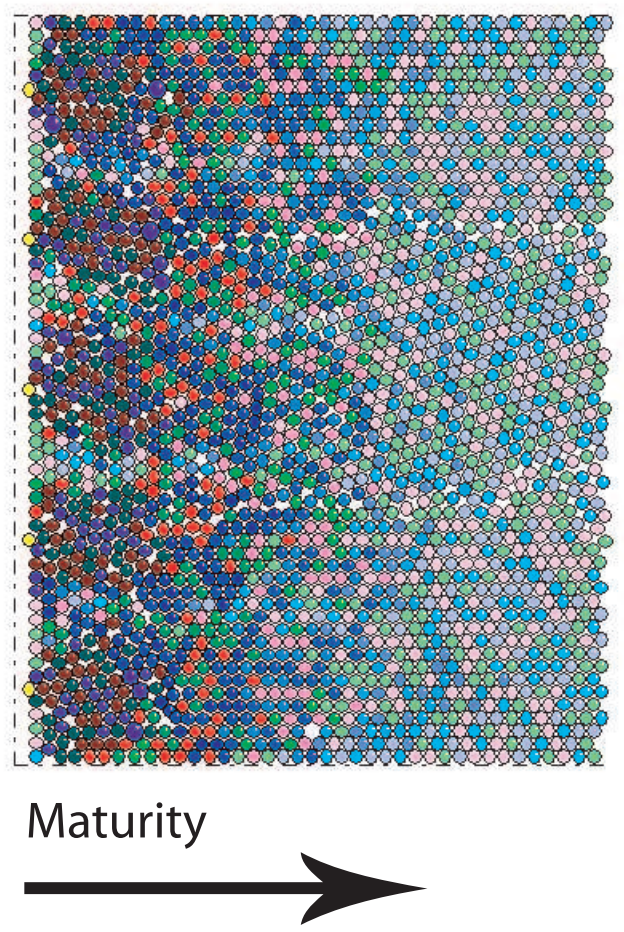

Figure 3: Example of normal hematopoiesis modeling. Yellow (stem) cells also called A0 can give birth to an other yellow stem cell, or one of the 3 cells (B1, E1, F1), being the origin of the 3 branches (red cells (red), white cells (blue) and platelets (green)respectively). E1 cells can give two sub-branches (which can be possible in the myeloid branch, when white cells split into neutrophil, basophil, and the eosinophil branches). These cells differentiate, die or leave the bone marrow with a certain rate. It is possible in this figure to see that the most mature cells (light colors) are on the right side of the screen. They correspond to the cells leaving the bone marrow as expected. 
right boundary. In other words, immature cells or blasts do not reach the blood vessels which corresponds to normal hematopoiesis. This option can be changed by the user, either by changing the box size representing the bone marrow, or by taking a different cell density (the cell density in figure 3 is 0.2 ).

Sometimes, some stem cells are not attached to the bone marrow. Thus, they can be gradually washed out. This phenomenon does not happen frequently in normal hematopoiesis, indeed, these cells are generally pressed to the left by other cells. However, if one consider a leukemic situation, this factor can appear to be essential. Indeed, remember in our example that stem cells are the only one able to self-renew. What would happen if we could allow some cells to have this property and decide not to attach them to the bone marrow. Then, they should differentiate like the other cells and be washed out from the bone marrow through the right boundary after a certain time. We show below that in this pathological case is not always as simple to predict as it seems.

In [8] the authors study this problem using a reaction-diffusion model taking the bone marrow porous structure into account. They give conditions under which it is possible to get the persistence of the pathology. The diffusivity property of the disease plays a crucial role there as well as the structure of the domain. Here, we do not have the porous structure, but we can change different parameters that lead to the same qualitative conclusions and explanations.

First of all, we need to describe properties of malignant cells in the software. It is known that leukemia can start with a single cell. Moreover, it can be any cell type $(A, B, C, .$. in the model). The cell type would determine then the nature of the leukemia. Malignant cells can be characterized by an excessive proliferation and by the absence (or deficiency) of differentiation which gives an abnormal high number of immature cells in the marrow first and the blood stream then.

We begin with the simplest scheme of leukemic hematopoiesis where each malignant cell gives two identical cells. Figure 4 shows the original malignant cell picked up by the user. The software allows the user to choose any cell and declare it malignant ( $X$ type). The properties of the malignant cells are indicated in the table in figure 4. In this particular case its proliferation rate is the same as for the stem cells.

Figures 5 and 6 show the time evolution of the same cell population. The region filled by black cells grows in all directions. At the first stage of the evolution leukemic and normal cells are practically separated in space. It is important to specify that production of normal cells does not decrease in the presence of leukemic cells. As assumed above, because the same cell flow is applied in the marrow from the left to the right the population dynamics should wash out leukemic cells. However, leukemic cells, since their number grows exponentially, create a strong local pressure that pushes other cells outside.

Therefore, there is a competition of two factors. On one hand, the cell flow that pushes leukemic cells out of the computational domain, on the other hand, the excessive pressure created by leukemic cells that allows them to spread in all directions and push other cell outside. At this stage, leukemic cells can leave the bone marrow to reach the blood vessels (figure 5, right) but immature normal cells do not reach the right boundary yet. In the next stage (figure 6, left), black cells fill an important part of the domain. The inflow of normal cells remains the same but they spend less time inside the marrow. As a result, immature normal cells leave it and can then 
Development of leukemia

\begin{tabular}{|c|c|c|c|c|c|c|c|c|c|}
\hline New & & & & & & & & & ?) $x$ \\
\hline & Parent & Chld1 & Chld2 & Chld3 & Chld4 & Time of.. & $+1-$ Tim. & Radius. & $\triangle$ \\
\hline$\checkmark$ & E1 & E2 & . & - & . & 200 & 40 & 0.01 & \\
\hline$\checkmark$ & E2 & E3 & . & . & . & 200 & 40 & 0.01 & \\
\hline$\checkmark$ & E3 & E4 & . & . & . & 200 & 40 & 0.01 & \\
\hline$\checkmark$ & E4 & . & . & . & . & $1 e+020$ & 2 & 0.01 & \\
\hline$\checkmark$ & F1 & F2 & . & . & . & 200 & 40 & 0.01 & \\
\hline$\checkmark$ & $\mathrm{F} 2$ & F3 & . & . & . & 200 & 40 & 0.01 & \\
\hline$\checkmark$ & F3 & . & .. & . & . & 200 & 40 & 0.01 & \\
\hline$\nabla$ & $\mathrm{F} 4$ & . & - & . & . & $1 e+020$ & 2 & 0.01 & \\
\hline$\checkmark$ & $x$ & $x$ & $x$ & - & . & 10 & 2 & 0.01 & \\
\hline$\square$ & & . & . & . & . & 0 & 0 & 0 & \\
\hline$\square$ & & . & . & . & . & 0 & 0 & 0 & \\
\hline$\square$ & & . & . & . & . & 0 & 0 & 0 & \\
\hline$\square$ & & . & . & . & . & 0 & 0 & 0 & \\
\hline$\square$ & & . & . & . & . & 0 & 0 & 0 & - \\
\hline$\square$ & & . & $\therefore$ & . & . & 0 & 0 & 0 & \\
\hline$\square$ & 1. & . & . & . & . & 0 & 0 & 0 & -1 \\
\hline$x=$ & $\longdiv { 0 . 4 }$ & & & & & & $c e=\max$. & & \\
\hline & $=0.6$ & & & Paint & & & & $=1$ & \\
\hline & New area & & 6 & Paint $\mathrm{AO}_{0}$ & $\Delta 1, \ldots$ & & & $=10$ & \\
\hline & ensity of $\mathrm{AO}$ & $0=0.2$ & $\sqrt{\checkmark}$ & $\mathrm{A} 0$ is fixe & $d \sqrt{v}$ & $f=a 4, g=0 / f$ & $g=b 4$ Pos & zapoleniija & \\
\hline $10=$ & 0 & $90=$ & 0 & & & & & & \\
\hline
\end{tabular}

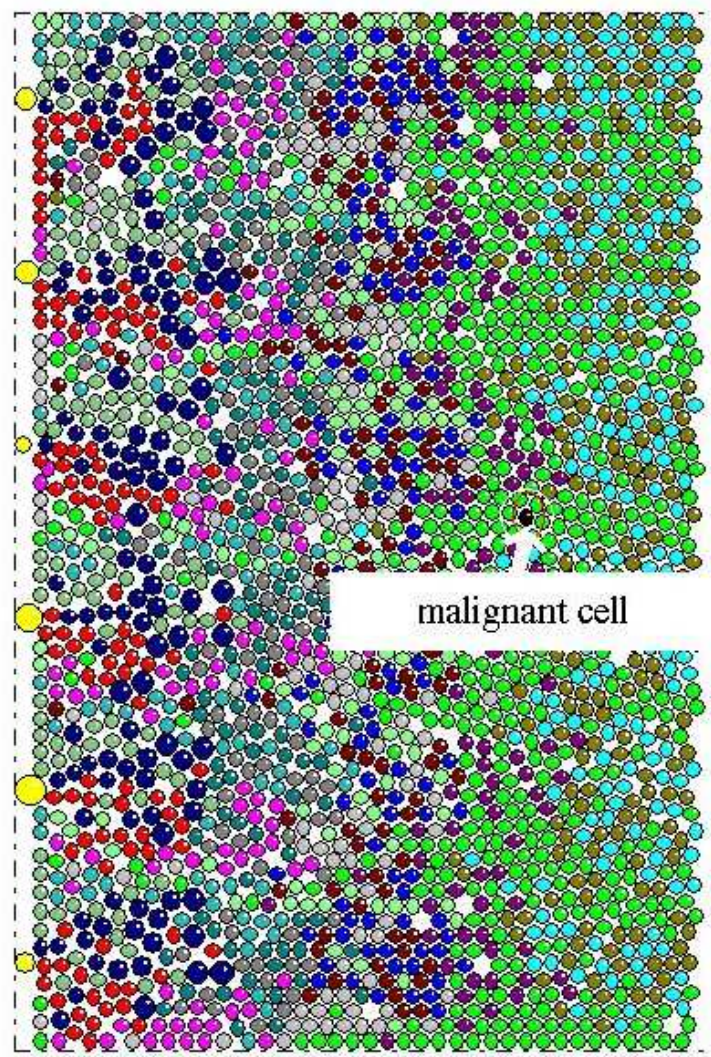

Figure 4: Similar figure as Figure 3, but in this case, we introduce a black (malignant) cell. 

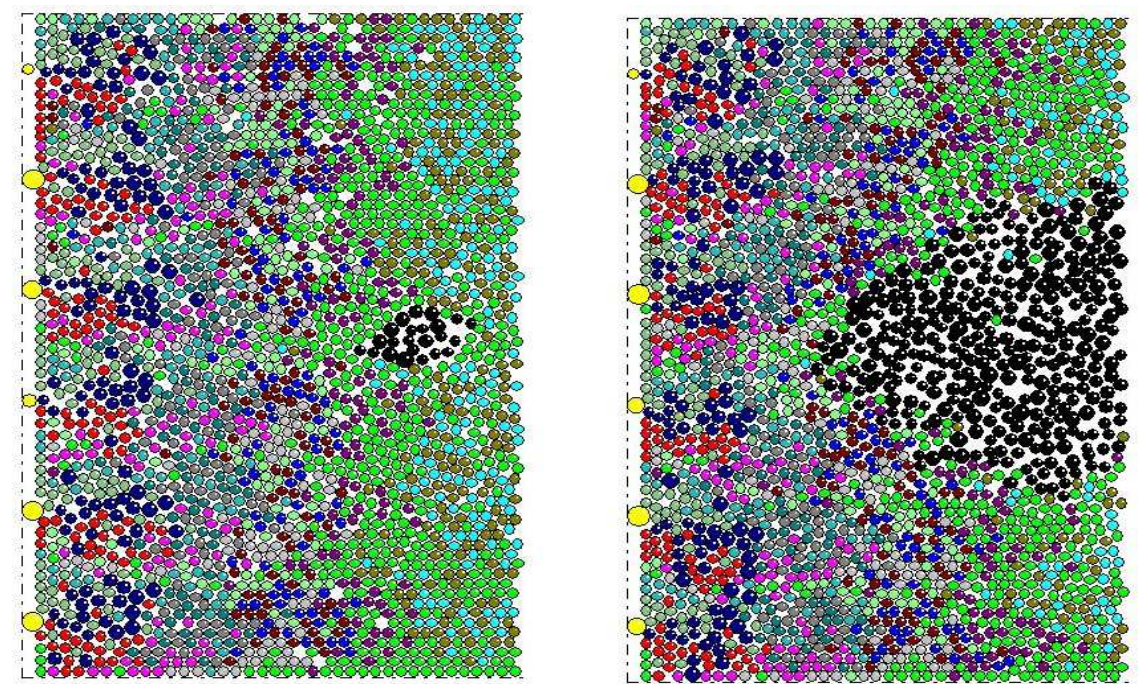

Figure 5: Example of the spread of the disease when the malignant (black) cells starting from a few group of malignant cells spread and start to wash out the healthy (colored) cells.
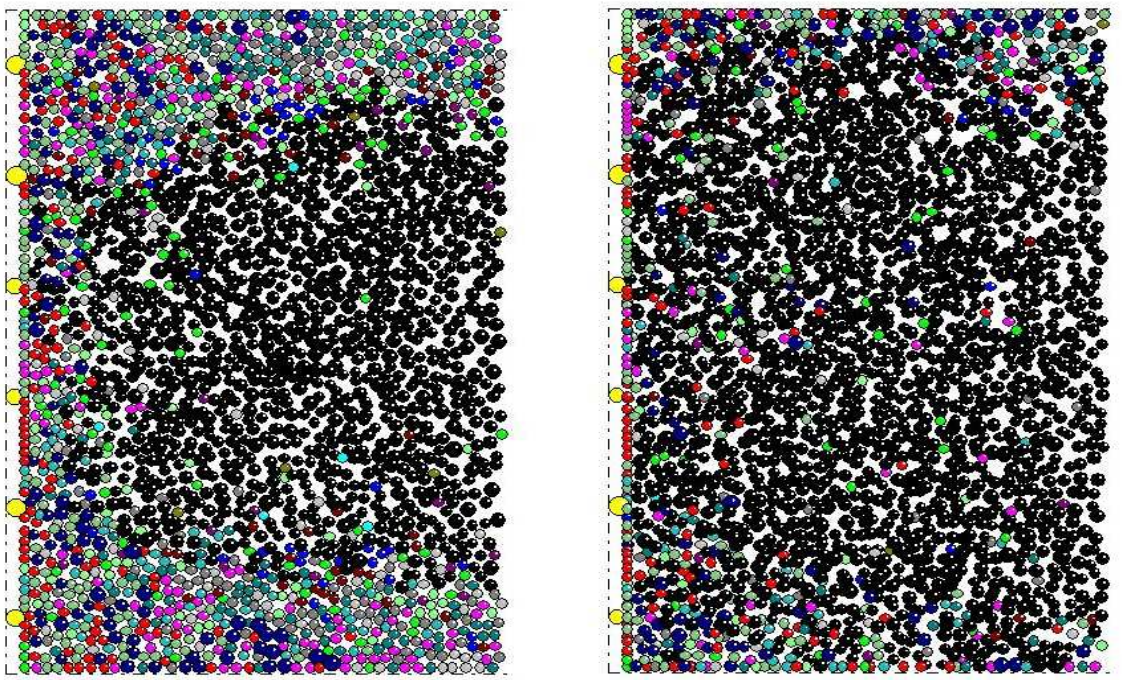

Figure 6: Last stage of the disease when almost all cells in the system are malignant (black) cells. 
be found in the blood stream. This is one of the main leukemia feature observed by the clinicians when diagnosing the disease. Thus, to summarize, two main factors contribute to the presence of blasts in blood vessels: first, a strong production of immature leukemic cells that do not differentiate because of some genetic mutations and second, and insufficient time left to normal cells for staying in the bone marrow.

After a certain time black cells fill the whole domain (figure 6, right). There is still a small quantity of normal cells, but they are rapidly pushed out of the domain.

It is clear that the possibility for leukemia to develop from a single malignant cell depends on the parameters we use. More specifically, it is related to the stem cells density and the proliferation rate of leukemic cells. In figure 7 for instance, we compare three simulations with different proliferation times for leukemic cells: the first one is $T_{X}=100$ time unit, the second 50 t.u., and the third 20 t.u. In each simulation, the cell distribution remains approximately constant with some possible oscillations around an average distribution (not shown here). For a sufficiently long proliferation time the region of black cells remains localized and does not spread to the whole domain (figure 7 left). On the contrary a rather short proliferation time would let the disease to spread quite fast (figure 7 right). On the other hand, if we increase the density of the stem cells and keep
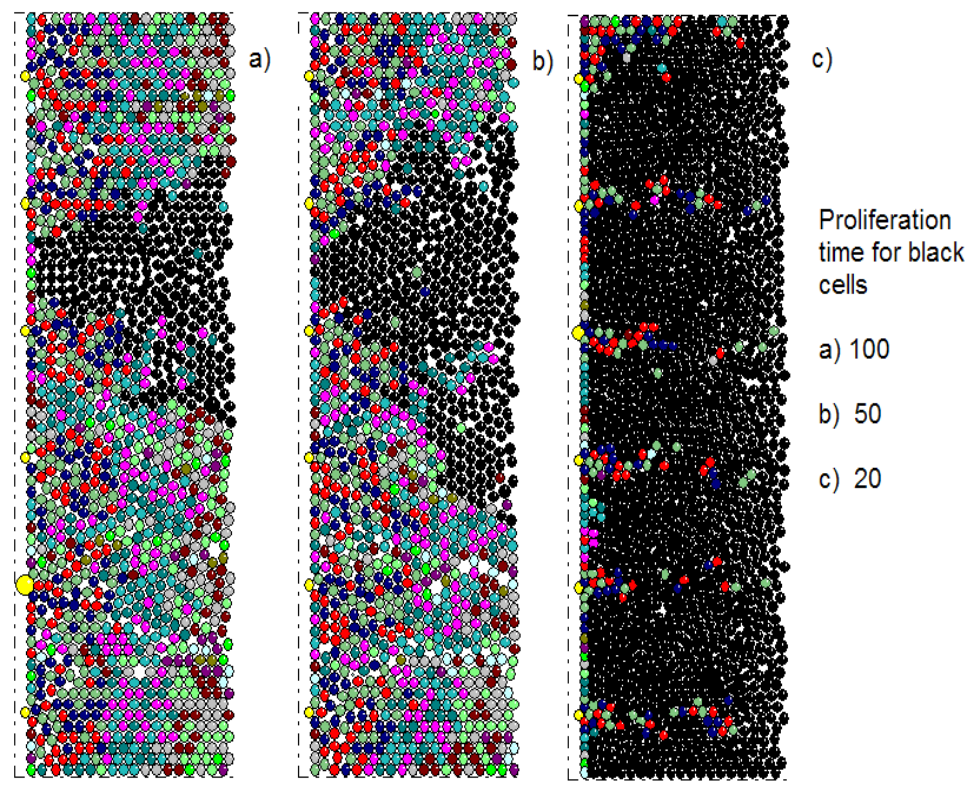

Figure 7: Comparison between three simulations with different proliferation times for leukemic cells and taken at different times. The first one is $T_{X}=100$ time unit, the second 50 t.u., and the third 20 t.u. In each case, the cell distribution remains approximately constant.

the same values for all other parameters, then leukemia has obviously less chances to develop (not shown here). It can also depend on the location of the original malignant cell. If this latter is closer to the right boundary of the domain (that is more mature), then black cells will have less time to multiply, and vice versa and each choice leads to a different type of leukemia as explained before. Let us detail now one of the most common, the so called chronic myelogenous leukemia (CML). 
In figure 8, we show a cell population at three different moments of time with the same parameters. The black cells form in here one, two or three domains, and these configurations repeat themselves approximately periodically in time. Concentrations of cells of all types oscillate in time as shown is the time series taken from this simulation in figure 9. This simulations has been done with arbitrary parameters not necessarily related to any real biological ones. However, the qualitative behavior is really close to some clinical data found in the literature (compare with figure 10 found in Fortin et al. [15]) and related to CML. This result appears really encouraging to us.
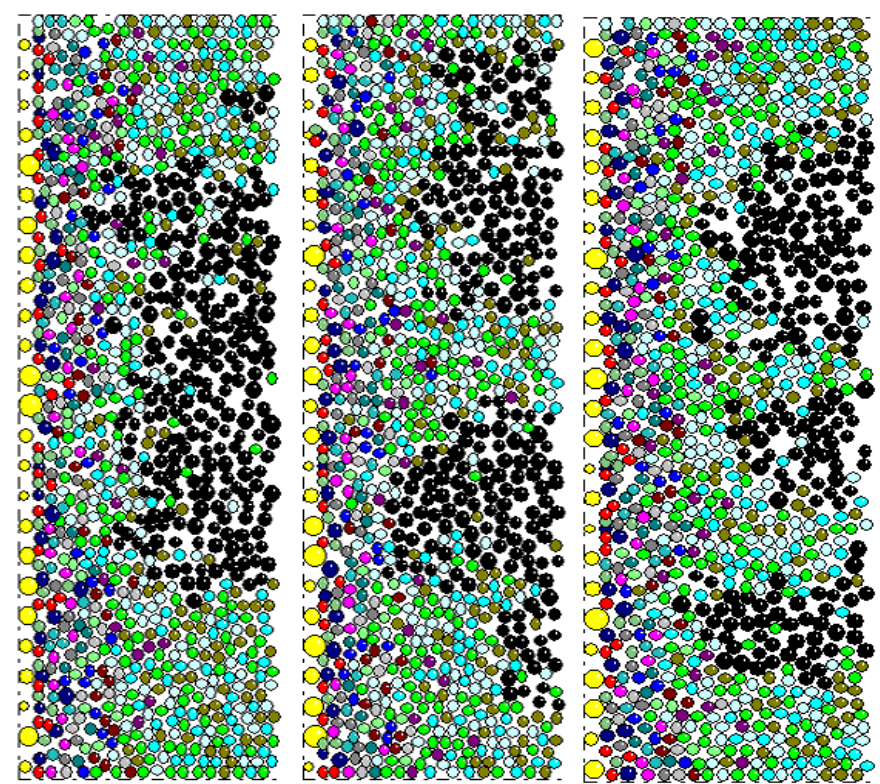

Figure 8: Cell distributions taken at three different times. The black cells form one, two or three domains. These configurations repeat approximately periodically in time (see the corresponding time series 9). It is possible to show that the concentrations of all cell types oscillate in time too.

In the last part of this section we simulate a more complex leukemic hematopoiesis. It is related to the so called leukemic stem cells. It is well known that not all leukemic cells have the same role in a tumor development (see [18] for instance). It is possible that, in a similar way as for stem cells in normal hematopoiesis some of leukemic cells can self-renew. This is what we consider in figure 11. We starts with yellow cells $X$, which self-renew and produce darker yellow cells $X_{1}$. These cells can also be self-produced or can give birth to black cells $X_{2}$, the mutant ones. Beginning from this stage the cells do not self-renew:

$$
\begin{gathered}
X \rightarrow X+X_{1}, \quad X_{1} \rightarrow X_{1}+X_{2}, \\
X_{2} \rightarrow X_{3}+X_{3}, \quad X_{3} \rightarrow X_{4}+X_{4}, \quad X_{4} \rightarrow X_{5} .
\end{gathered}
$$

If we characterize leukemic stem cells by their ability to self-renew, then yellow cells $X$ and $X_{1}$ correspond to these kind of cells while black cells $X_{2}, X_{3}, X_{4}$, and $X_{5}$ are leukemic cells not able 


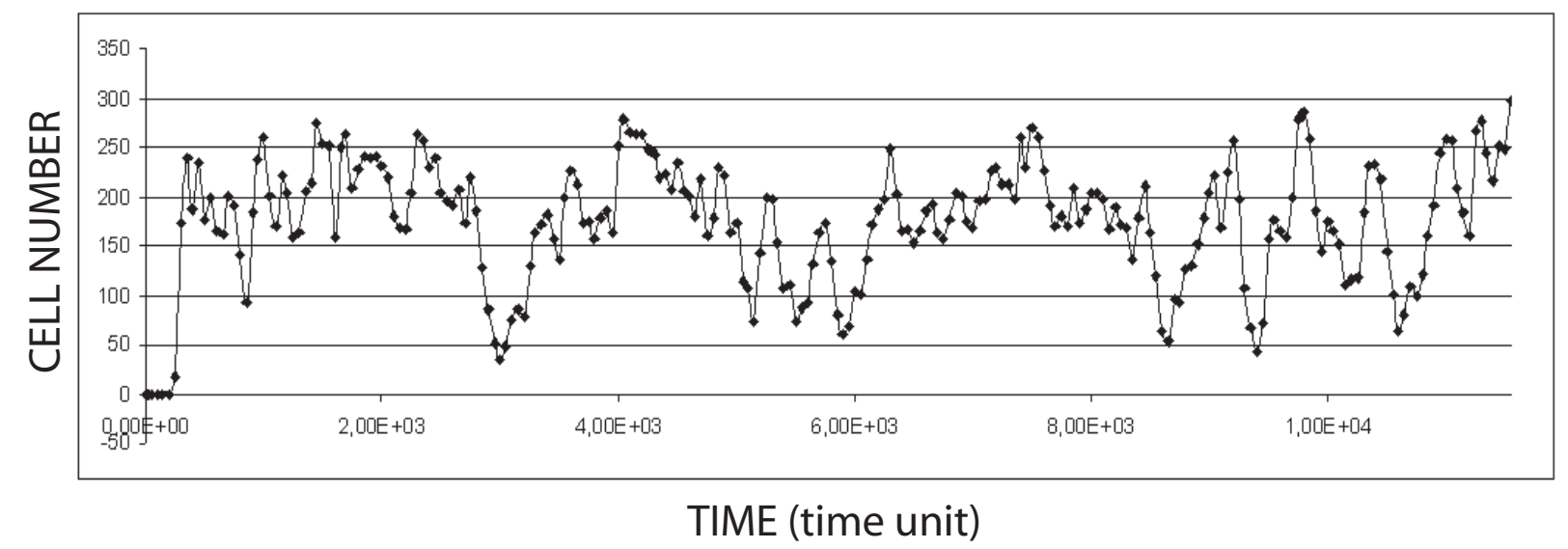

Figure 9: Time series of the malignant cell population in the bone marrow. This case could correspond to a CML case.

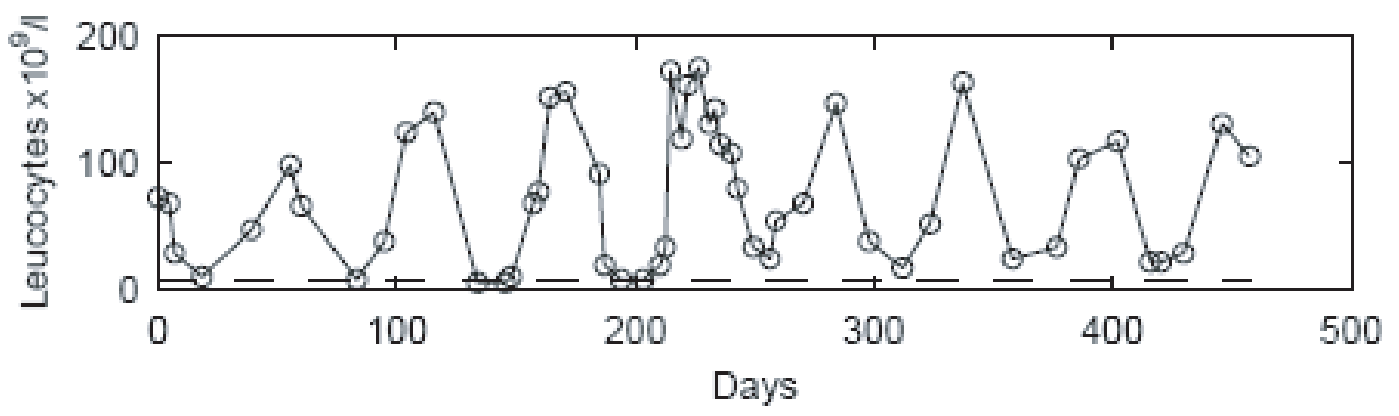

Figure 10: Time series of a leukocyte population in the bone marrow taken from a patient having CML. Real data taken from Fortin et al. [15]. 
Leukemia stem cells
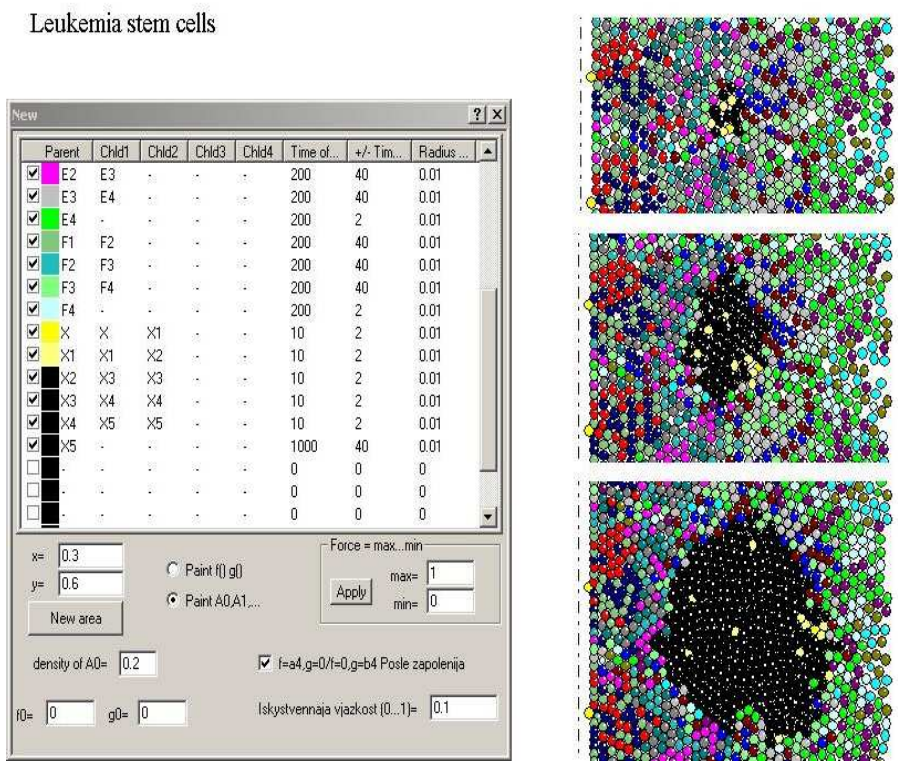

Figure 11: First part: evolution of the disease spread. The malignant (black) cells are growing from a malignant focus but not for a long time (see the second part in figure 12)

to renew themselves. Figure 11 (right) and figure 12 show the time evolution of the system. In the beginning, the number of black cells increases rapidly, and their region has clearly identified boundaries with no normal cells inside. After some time this region reaches the right boundary of the domain (which is the "entrance" to the blood circuit) and some black cells leave it. Then after, the black region decreases, splits and disappear being washed out by the healthy cells.

A question could arise then here. Why after some period of rapid development leukemia cells would completely disappear from the computational domain? The answer to this question can be given on the basis of observations of individual cells. Each cell, independently of its type (except for normal stem cells attached to the left boundary) leaves the computational domain after a certain amount of time. This is not a rigorous mathematical result but a conjecture confirmed by the numerical simulations. Yellow cells $X$ too are involved in this dynamics in the sense that since they renew themselves without increasing their number, their loss cannot be compensated and consequently their number decreases in time. This is why after a certain time, leukemic cells disappear completely. One could consider also the following scheme;

$$
X \rightarrow X+X_{1}, \quad X_{1} \rightarrow X+X_{2}, \quad X_{2} \rightarrow X_{3}, \quad X_{3} \rightarrow X_{4}
$$

where the number of $X$ cells increases in time, providing a very likely leukemia development (not shown here). 


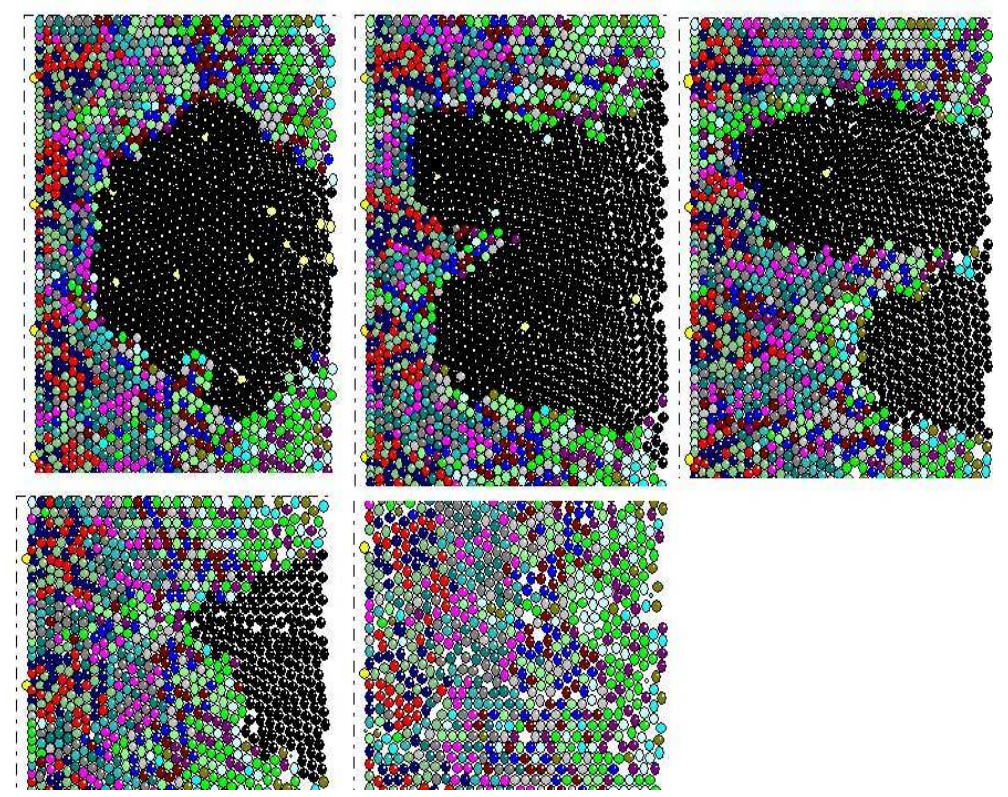

Figure 12: Second part: the malignant (black) cells keep on spreading but finally they get washed out by the healthy cells. The disease free steady state is then stable in this example.

\section{Cell communication}

Hematopoiesis is controlled by a complex system of external and internal feedbacks most of the time by hormone stimulations. It is believed that blood cells in the bone marrow produce some biochemical products called growth factors that can influence their dynamics. More particularly they could influence the differentiation choices for undifferentiated cells. This has not been proven yet, and it is still an open question. Some biologists on the contrary suppose that this process should be stochastic only. That is a stem cell would produce a differentiated cell with a certain probability that depends only on the the cell itself. To the best of our knowledge, the exact mechanism of differentiation is not known yet. However, it is interesting to anticipate this hypothesis with our software. On one hand, the stochastic point of view for differentiation, this work is still under investigation as we mentioned it in the previous sections. On the other hand, the cell communication hypothesis involving some external stimulations is complete and this is what we develop here. Let us consider the simplest scheme

$$
A \rightarrow A+B, A \rightarrow A+C
$$

It is possible to complicate this process, but we refer the user to the manual for more complex cases. Here, we specify which $A$-cells choose the first path, that is the B-type, and which ones the second, that is the C-type. We consider here three cell types: undifferentiated white cells and differentiated red or blue cells ( see figure 13). Each cell is characterized by two parameters, $f$ and $g$ such that the $i$-th cell is associated with the functions $f_{i}(t)$ and $g_{i}(t)$. We suppose that when a new cell appears, it is undifferentiated and we put for it $f=f_{0}, g=g_{0}$. Time evolution of the 
Cell communication

\section{f5, g5}

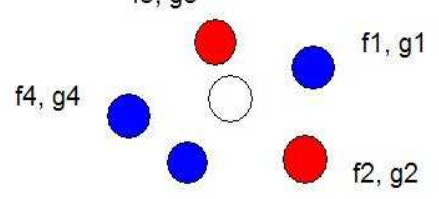

f3, g3

1. Cell childhood (what colour to choose?)

$d f / d t=a(F-f), d g / d t=a(G-g)$

$\mathrm{F}=$ average of $\mathrm{fi}, \mathrm{G}=$ average of $\mathrm{gi}$

Critical condition: $f=f^{*}$ or $g=g^{*}$
2. Adult life

Blue: $\quad d f / d t=P\left(f, g^{*}\right), g=g^{*}$

Red: $\quad d g / d t=Q\left(f^{*}, g\right), f=f^{\star}$

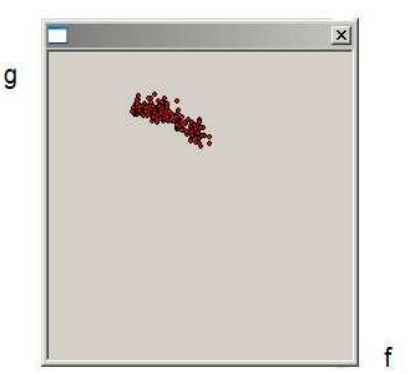

Figure 13: Illustration of the cell communication modeling. Each cell "i" at a time $t$ is characterized by two parameters, $f_{i}(t)$ and $g_{i}(t)$. Under specific conditions the neighboring cells can influence the undifferentiated (white) ones. And the influence of the neighbors depends on the numbers of each cell type around. Here there are more blue than red cells surrounding the white cell, and thus, this latter will differentiate into a blue one. After a certain time, we stop the simulation, we count the number of each cell type ( $f$ and $g$ ) plot their distribution in the $f-g$ plane. The functions $\mathrm{P}$ and $\mathrm{Q}$ are defined below in this section, and $a$ is a constant parameter. 
functions $f_{i}$ and $g_{i}$ for an undifferentiated cell is given by the equations

$$
\frac{d f_{i}}{d t}=a\left(F_{i}-f_{i}\right), \quad \frac{d g_{i}}{d t}=a\left(G_{i}-g_{i}\right)
$$

where $a$ is a constant,

$$
F_{i}=\sum_{j \neq i} f_{j}, \quad G_{i}=\sum_{j \neq i} g_{j}
$$

and the sum is taken over closest neighbors. Equation (3.1) has the following meaning. Each cell sends out or loses the molecules $f$ and $g$ with the rate proportional to their concentration inside the cell. It also receives the ones coming from the neighboring cells with the rate proportional to their concentrations inside the neighboring cells. A white cell remains undifferentiated while

$$
f_{i}^{2}(t)+g_{i}^{2}(t) \leq \sigma
$$

where $\sigma$ is a given parameter. In other words, when the concentrations of $f$ and $g$ becomes sufficiently high, the cell chooses its type. If $f$ is greater than $g$ at this moment, then it becomes red, otherwise blue (with the color code chosen here).

Once the differentiation occurs and the cell chooses its type, further evolution of $f$ and $g$ becomes different. For red cells

$$
\frac{d f_{i}}{d t}=P\left(f_{i}, g_{i}\right), \quad g_{i}=g_{i}^{*}
$$

for blue cells

$$
\frac{d g_{i}}{d t}=Q\left(f_{i}, g_{i}\right), \quad f_{i}=f_{i}^{*},
$$

where $f_{i}^{*}$ and $g_{i}^{*}$ are the values of $f$ and $g$ at the moment of the cell differentiation. After differentiation, the value of $f$ in red cells increases, the value of $g$ remains constant; for blue cells $g$ increases, $f$ remains constant. This means that the cell produces bio-chemical substances according to its type. We consider quadratic functions $P$ and $Q$ :

$$
P(f, g)=a_{1}+a_{2} f+a_{3} f^{2}+a_{4} f g+a_{5} g, \quad Q(f, g)=b_{1}+b_{2} g+b_{3} g^{2}+b_{4} f g+b_{5} f,
$$

where $a_{i}$ and $b_{i}$ are some constants. This functions are chose to be quadratic functions arbitrarily for the sake of simplicity. But, we can keep in mind that this can be changed anytime, depending on biological hypotheses. We describe now the numerical simulations shown in figure 15. Stem cells are undifferentiated and located as before at the left boundary. They produce undifferentiated cells with the initial values $f=f_{0}, g=g_{0}$. When the whole domain is filled with undifferentiated cells, the simulation is stopped. Each cell is being prescribed one of the two types, red or blue (with some given values $f$ and $g$ ) in a random way. After that the simulation starts again. New undifferentiated cells are now surrounded by differentiated cells and are committed to choose their type. After some time, some regions filled by red and blue cells begin to appear. Figure 15 (center) shows a layered structure after some time from an initial random distribution. This structure is not stationary, the layers can move, appear and disappear depending on the parameters chosen. 


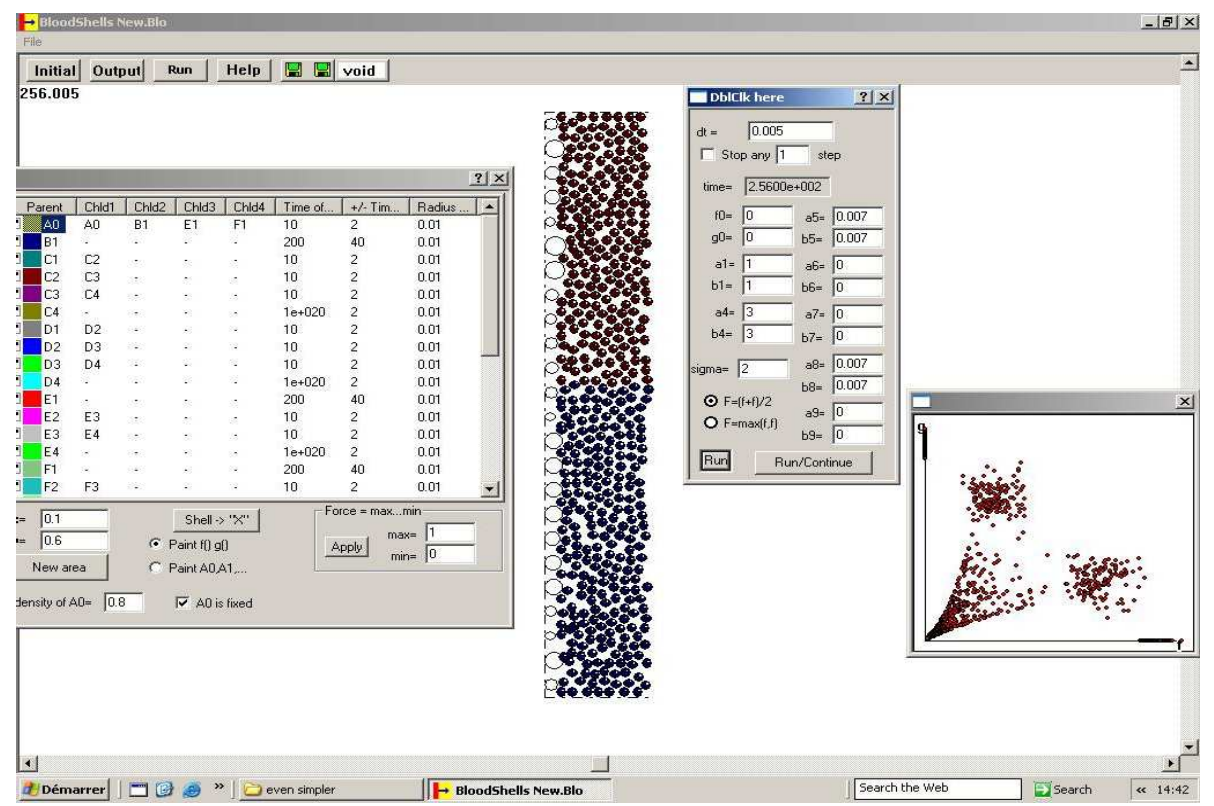

Figure 14: Simulation when the production of $f$ and $g$ substances is not sufficient. After some time, the system loses its differentiation and so the points in the $f-g$ plane converge to the origin.

Indeed, since the initial distribution is random, two different simulations with the same values of parameters can give different results. In some cases, only one of two cell types remain, and another disappear completely. As it is explained above, each cell is characterized by two functions $f_{i}(t)$ and $g_{i}(t)$ that determine its type and its color. Let $\tau_{i}$ be the moment of time when the $i$-th cell leaves the computational domain (that is the bone marrow for us). We plot then the point $\left(f_{i}\left(\tau_{i}\right), g_{i}\left(\tau_{i}\right)\right)$ in the $(f, g)$-plane. If we do it for each cell that leaves the domain, then we can characterize the population of cells that reaches the blood stream. Figure 15 (right) shows two clouds of points, one of them corresponds to red cells, another one to blue cells. From the biological point of view, this could correspond to formation of cell colonies in the bone marrow which is a well known phenomenon though its mechanism is still unclear. As said before, the structures of differentiated cell colonies depend on the parameters set up in the problem. Namely, differentiated cells should produce a sufficient amount of substances $f$ and $g$ to maintain the system of cells in differentiated state. Otherwise the system can become undifferentiated as shown in figure 14. In this figure, at the first stage, we observe the emergence of some structures for the differentiated cells. There are two clouds of points in the $(f, g)$-plane. After some time, the system of cells loses its differentiation. The points at the $(f, g)$-plane converge then to the origin.

We describe finally how leukemia can influence the structured populations in the cell communication case. Let us consider a structured population that appears after some time from a random initial distribution (figure 16, left). We introduce a single (blue) leukemic cell assuming that the values $f$ and $g$ for this cell are constant (independent of time). It proliferates producing each time two identical cells with the same values of $f$ and $g$. Multiplication of leukemic cells acts in two ways. First of all, red cells are pushed out of the domain because of the mechanical interaction. 


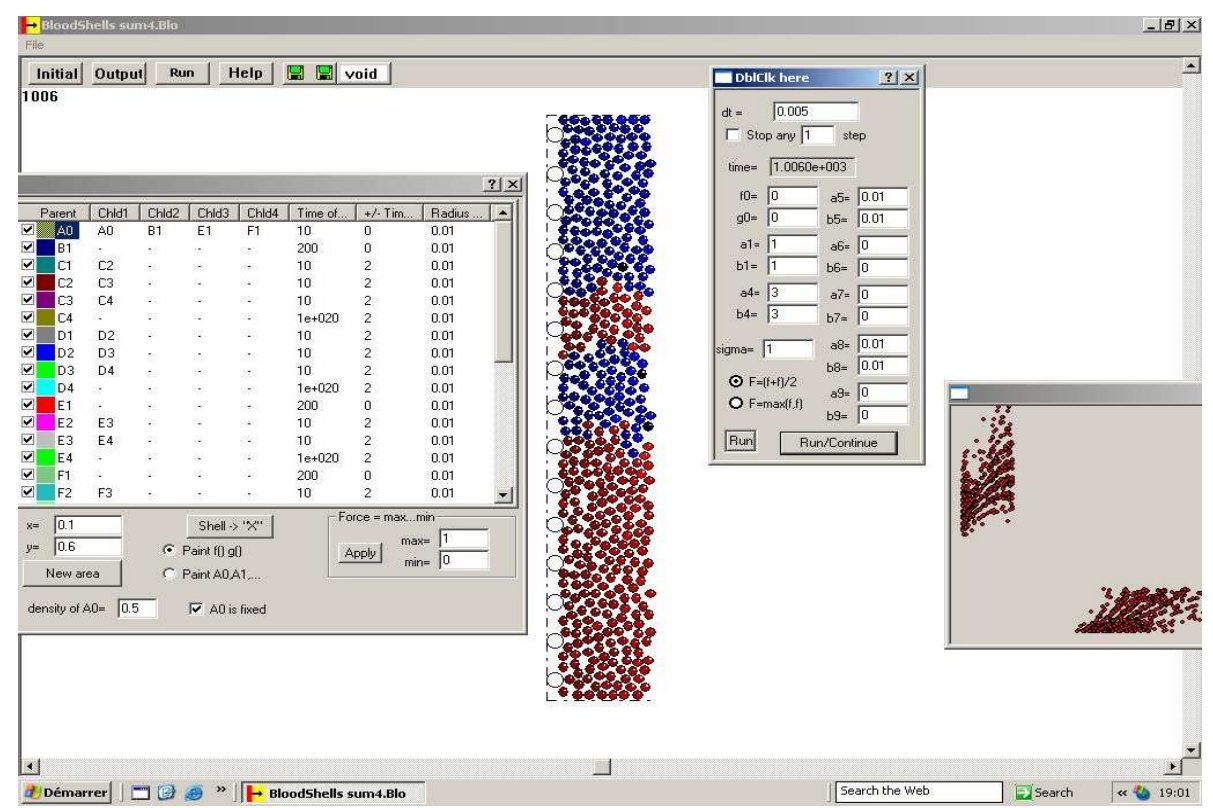

Figure 15: Simulation of a case where stem cells are undifferentiated and located at the left boundary. They produce undifferentiated cells with the initial values $f=f_{0}, g=g_{0}$. Once the whole domain is filled with undifferentiated cells, the simulation is stopped. Each cell is being prescribed randomly one of two types, red or blue (with some given values $\mathrm{f}$ and $\mathrm{g}$ ). Then simulation starts again. New undifferentiated cells are now surrounded by differentiated cells and can choose their type. After some time, some regions filled by red and blue cells start to appear. A layered structure appears after some time from an initial random distribution. The distribution is then plotted in the $f-g$ plane. 


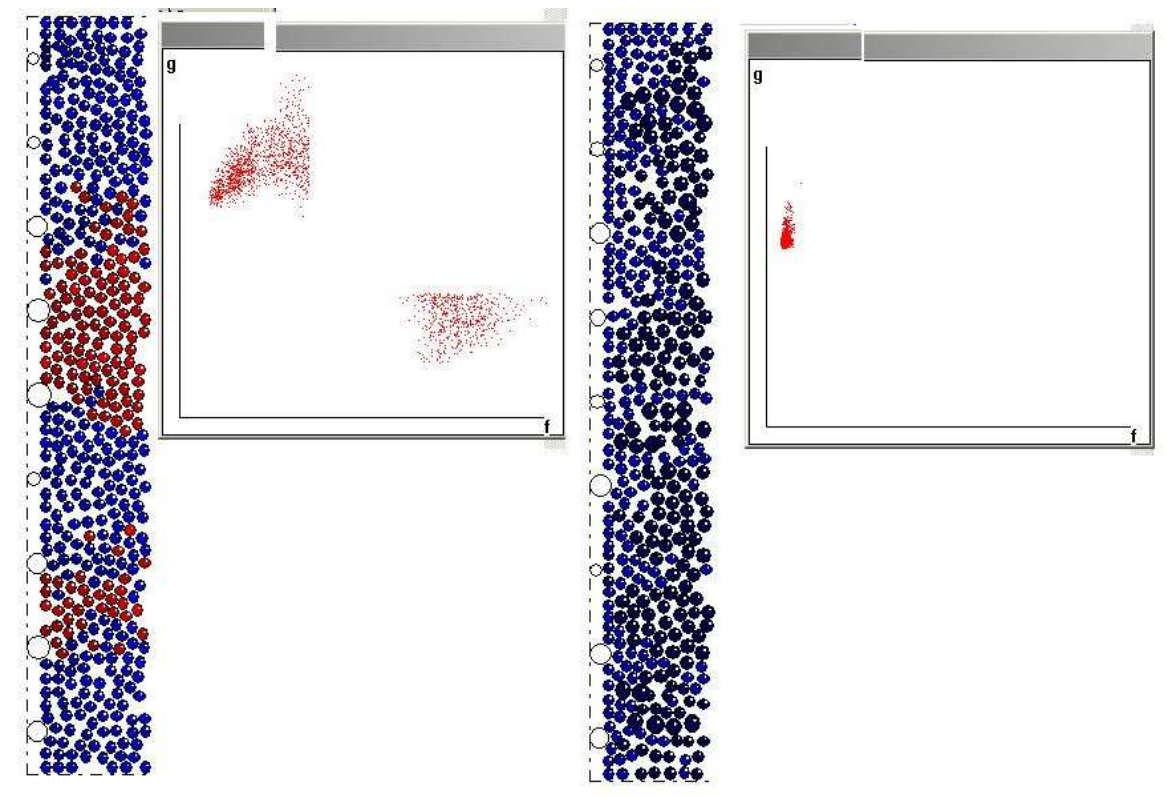

Figure 16: Simulation of the case where leukemic (blue) cells appear and wash out all the healthy (red) cells. The final distribution in the cloud is then exclusively about the $g$ cells.

Second, differentiation of new normal cells occurs basically in the direction of blue cells because there are more and more of these latter in the computational domain. As a result of this evolution we have a strong change in the $(f, g)$-representation. Instead of two clouds of points (left) we have only one strongly localized cloud: the malignant cell type. We remark that this representation of cells in the $(f, g)$-plane is strongly related to characterization of cells with flow cytometry. But this aspect, as well as comparing clinical data using the CFSE (CarboxyFluorescein diacetate Succinimidyl Ester) cell marker with our approach and the one introduced in [7] will be deeply investigated in a future work.

\section{Conclusion}

Our goal here was to give a representation of hematopoiesis using a software based on a simple model. We believe that this could be an excellent interface between biologists and mathematicians. The software can be modified very easily by adding new conditions, some more complex equations and more parameters depending on the expectations of biologists and clinicians. It has been shown here, that it is possible to simulate normal hematopoiesis as well as different schemes of leukemia like chronic myelogenous leukemia. All this simulations here, so far, have been done from a qualitative point of view first. The quantitative representations still needs many feedbacks between us and experimental biologists. By some changes in the parameters, it would be possible to simulate any other periodic hematological diseases like cyclical neutropenia or thrombocytopenia for instance. We showed here the important role played by stem cells. We also proved that 
the pressure occurring in the bone marrow under the influence of high proliferation of malignant cells could give an explanation regarding the possible disappearance of the disease, the appearance of periodic behavior, or the dramatic spread of the malignant cells. Finally, we assume that cell communication is possible, and the influence of the cell neighborhood is quite important. This has not been proven yet biologically, but could be an explanation for the brilliant recovery of the system suffering from an important blood loss. The influence of growth factor would play the most important role under this circumstance, and could be supported by cell communication. As we have shown here, this could be also a disadvantage under the presence of malignant cells. These cells could influence the others more rapidly.

Our next objective will be to validate our results with clinicians and biologists. It would be interesting to show the quantitative behavior of the cyclical diseases in time series figures. It would be also important to modify the software in such a way that stem cells can give birth to the different types of progenitor cells in a specific probability that could be changed by the user. Finally, as mentioned in the previous section, we would like to compare our results with the ones found by Bernard et al. in [7] that is the behavior of cell generations measured with a cell marker called CFSE. All this will be presented in future works.

\section{References}

[1] M. Adimy, F. Crauste, S. Ruan. Periodic Oscillations in Leukopoiesis Models with Two Delays. J. Theor. Biol., 242 (2006), 288-299.

[2] M. Adimy, F. Crauste, S. Ruan. A mathematical study of the hematopoiesis process with applications to chronic myelogenous leukemia. SIAM J. Appl. Math., 65 (2005), No. 4, 13281352.

[3] M. Adimy, L. Pujo-Menjouet. A singular transport model describing cellular division. C.R. Acad. Sci. I-Math, 332 (2001), 1071-1076.

[4] M. Adimy, L. Pujo-Menjouet. Asymptotic behavior of a singular transport equation modelling cell division. Discrete Contin. Dyn. Syst. Ser. B, 3 (2003), No. 3, 439-456.

[5] S. Bernard, J. Bélair, M.C. Mackey. Oscillations in cyclical neutropenia: New evidence based on mathematical modeling. J. Theor. Biol., 223 (2003), 283-298.

[6] S. Bernard, J. Bélair, M.C. Mackey. Bifurcations in a white-blood-cell production model. C. R. Biologies, 327 (2004), 201-210.

[7] S. Bernard, L. Pujo-Menjouet, M.C. Mackey. Analysis of cell kinetics using a cell division marker: Mathematical analysis of experimental data, Biophy. J., 84 (2003), 3414-3424.

[8] N. Bessonov, A. Ducrot, V. Volpert. Modelling of Leukemia Development in the Bone Marrow, Proc. of the Annual Symposium on Math. Applied in Biology and Biophysics, XLVIII, 2 (2005), No. 48, 79-88. 
[9] F.J. Burns, I.F. Tannock. On the existence of a $G_{0}$ phase in the cell cycle. Cell Tissue Kinet., 19 (1970), 321-334.

[10] C. Colijn, M.C. Mackey. A mathematical model of hematopoiesis: Periodic chronic myelogenous leukemia, part 1. J. Theor. Biol., 237 (2005), 117-132.

[11] F. Damiola, C. Keime, S. Gonin-Giraud, S. Dazy, O. Gandrillon. Global transcription analysis of immature avian erythrocytic progenitors: from self-renewal to differentiation. Oncogene, 23 (2004), 7628-7643.

[12] S. Dazy, F. Damiola, N. Parisey, H. Beug, O. Gandrillon. The MEK-1/ ERKs signalling pathway is differentially involved in the self-renewal of early and late avian erythroid progenitor cells. Oncogene, 22 (2003), 9205-9216.

[13] J. Dyson, R. Villella-Bressan, G.F. Webb. A singular transport equation modelling a proliferating maturity structured cell population. Can. Appl. Math. Quart., 4 (1996), 65-95.

[14] J. Dyson, R. Villella-Bressan, G.F. Webb. A singular transport equation with delays. Int. J. Math. Math. Sci., 6 (2003), No. 32, 2011-2026.

[15] P. Fortin, M.C. Mackey. Periodic chronic myelogenous leukemia: Spectral analysis of blood cell counts and etiological implications. Brit. J. Haematol., 104 (1999), 336-345.

[16] C. Haurie, D.C. Dale, M.C. Mackey. Cyclical neutropenia and other periodic hematological diseases: A review of mechanisms and mathematical models. Blood, 92 (1998), 2629-2640.

[17] T. Hearn, C. Haurie, M.C. Mackey. Cyclical neutropenia and the peripheral control of white blood cell production. J. Theor. Biol., 192 (1998), 167-181.

[18] J.P. Huntly, D.G. Gilliland. Leukemia stem cells and the evolution of cancer-stem-cell research. Nature Reviews. Cancer, 5 (2005), 311-321.

[19] M.J. Koury, M.C. BOondurant. Erythropoietin retards DNA breakdown and prevents programmed death in erythroid progenitor cells. Science, 248 (1990), No.4953, 378-381.

[20] L.G. Lajtha. On DNA labeling in the study of the dynamics of bone marrow cell populations, in: Stohlman, Jr., F. (Ed), The Kinetics of Cellular Proliferation, Grune and Stratton, New York, pp. 173-182, 1959.

[21] M.C. Mackey. Cell kinetic status of hematopoietic stem cells, Cell Prolif., 34 (2001), 71-83.

[22] M.C. Mackey, C. Ou, L. Pujo-Menjouet, J. Wu. Periodic oscillations of blood cell populations in chronic myelogenous leukemia, SIAM J. Math. Anal., 38 (2006), No. 1, 166-187.

[23] L. Pujo-Menjouet, S. Bernard, M.C. Mackey. Long period oscillations in a $G_{0}$ model of hematopoietic stem cells. SIAM J. Appl. Dynam. Syst., 4 (2005), No. 2, 312-332. 
[24] L. Pujo-Menjouet, M.C. Mackey. Contribution to the study of periodic chronic myelogenous leukemia. C. R. Biologies, 327 (2004), 235-244.

[25] M. Santillan, J. Blair, J.M. Mahaffy, M.C. Mackey. Regulation of platelet production: The normal response to perturbation and cyclical platelet disease. J. Theor. Biol., 206 (2000), 585-903.

[26] J. Swinburne, M.C. Mackey. Cyclical thrombocytopenia: Characterization by spectral analysis and a review. J. Theor. Med., 2 (2000), 81-91.

[27] F.M. Watt, B.L. Hogan. Out of Eden: stem cells and their niches. Science, 287 (2000), 14271430 .

\section{A The model behind the software}

Each cell is considered as a disk in the plane. Cells of different types are shown with different colors (figure 17). A cell behavior is characterized by its interactions with other cells, its proliferation, differentiation and apoptosis properties. All this is explained below.

1. Mechanical interaction. Interaction of two neighboring cells is determined by the interaction potential. The sum of forces acting on each cell from other cells determines the cell motion according to Newton's law with a possible damping because of the friction with other cells. Thus, we use an approach similar to molecular dynamics simulations even though the potential is different. We have

$$
x_{i}^{\prime \prime}-\epsilon x_{i}^{\prime}+\frac{1}{m} \sum_{i \neq j} f_{i j}=0,
$$

where $x_{i}$ is the coordinate of the center of the $i$-th cell, $m$ is its mass, $\epsilon$ is the damping coefficient, $f_{i j}$ is the force acting between the cells $i$ and $j$. We put

$$
f_{i j}=-\phi\left(\left|x_{i}-x_{j}\right|\right)
$$

where the function $\phi(r, t)$ equals zero for $r \geq r_{i}+r_{j}$ and it goes to infinity as $r$ decreases. Here $r_{i}$ and $r_{j}$ are the radii of the cells $i$ and $j$, respectively (which can depend on time). Thus, two cells push each other when the distance between their centers is less than the sum of their radii.

2. Chemical interaction. Cells can produce bio-chemical compounds called growth factors. They can influence behavior of other cells: apoptosis [19] and possibly differentiation though this still unclear from the biological point of view. We shall carry out cell modeling taking chemical interactions into account. These interactions are discussed below.

3. Cell properties. There can be different cell types in the model. For each type, the user prescribes its behavior, namely, its lifetime and the type of its offsprings in the case of proliferation or differentiation. Let us denote for instance the cell types by $A, B, C$ here. For each of them a specific lifetime $T_{A}, T_{B}, T_{C}$ is respectively prescribed. Actually, it is interesting to note that each 
lifetime is not prescribed exactly but with a random interval around its average value. For example, for cells of the type $A$ it is $\left[T_{A}-\tau_{A}, T_{A}+\tau_{A}\right]$ with an equal probability inside this interval. When the lifetime of a given cell is over, three possibilities are offered to it:

1. it dies, that is the corresponding circle is removed from the computational domain (gradually in time, as shown in figure 17 ('apoptosis' arrow) ),

2. it differentiates, that is the cell type is changed to another one without changing cell position (it is represented here by a color change)

3. it proliferates, that is the cell is replaced by two other cells. The types of new cells are prescribed by the user. The mother cell grows before dividing, the area of the corresponding circle equals the sum of areas of new circles after the division (this is shown in figure 17 ('stem cell about to divide' arrow) .

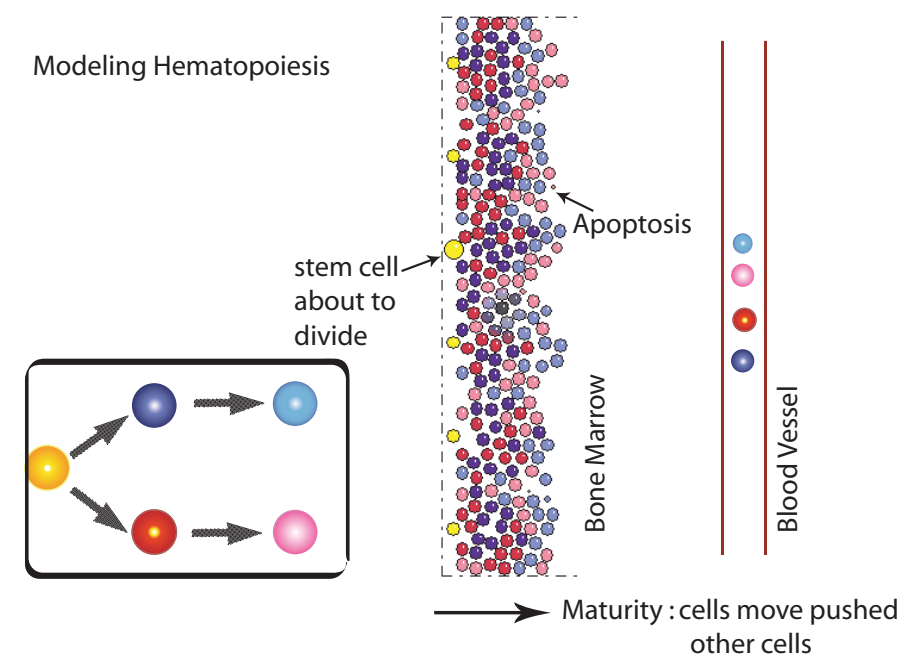

Figure 17: Example of what could be considered by the software. The yellow disks represent stem cell, red and blue cells direct offsprings of the yellow cells, and green their secondary offspring -or also direct offsprings of the red cells-. Yellow (stem) cells are attached to the wall on the left, and all the other cells are pushed away to the right by division, and released in the blood systems.

Because the aim of our software was initially to simulate hematopoietic cells in the bone marrow, the example we introduce below corresponds to blood cell proliferation. It is given in the table of figure 3. The user of the software can feel free to change these conditions depending on what is investigated. 
The software allows the introduction up to four daughter cells after one division, for example,

$$
A \rightarrow A+B+C+D,
$$

where $A$ could represent the stem cell, B,C,D the three different lineages (erythrocytes, leukocytes and platelets). Though the cases with three or four cells are not realistic from a biological point of view, it can be convenient for the modeling. We are currently working on a new version of the software where stochasticity occur at the first division of a stem cell. There would be a different probability for this cell to give birth to two stem cells, or either one stem cell and in particular, the last example can be considered as an approximation of the scheme

$$
A \rightarrow A+B, A \rightarrow C+D
$$

The scheme (A3) implies the introduction of probabilities for each of the two divisions, $A \rightarrow A+B$ and $A \rightarrow C+D$. However, in this work we do not introduce stochastic cell division (it will be considered in the subsequent work) 